\title{
Solitary infantile choriocarcinoma of the liver: MRI findings
}

\author{
van der Hoef, Marianne ; Niggli, Felix K ; Willi, Ulrich V ; Huisman, Thierry A G M
}

\begin{abstract}
Infantile hepatic choriocarcinoma is a rare, highly malignant germ-cell tumour believed to result from a choriocarcinoma of the placenta that spreads to the child. Most infants present with a characteristic clinical picture of anaemia, hepatomegaly and precocious puberty. Imaging findings, including conventional MRI, may be non-specific. To improve the accuracy of diagnosis, we present the imaging findings of contrast-enhanced dynamic MRI in a 4.5-month-old boy with infantile hepatic choriocarcinoma
\end{abstract}

DOI: https://doi.org/10.1007/s00247-004-1212-x

Posted at the Zurich Open Repository and Archive, University of Zurich

ZORA URL: https://doi.org/10.5167/uzh-156263

Journal Article

Published Version

Originally published at:

van der Hoef, Marianne; Niggli, Felix K; Willi, Ulrich V; Huisman, Thierry A G M (2004). Solitary infantile choriocarcinoma of the liver: MRI findings. Pediatric Radiology, 34(10):820-823.

DOI: https://doi.org/10.1007/s00247-004-1212-x 


\author{
Marianne van der Hoef \\ Felix K. Niggli \\ Ulrich V. Willi \\ Thierry A. G. M. Huisman
}

\section{Solitary infantile choriocarcinoma of the liver: MRI findings}

Received: 1 March 2004

Revised: 23 March 2004

Accepted: 28 March 2004

Published online: 28 July 2004

(C) Springer-Verlag 2004

\begin{abstract}
Infantile hepatic choriocarcinoma is a rare, highly malignant germ-cell tumour believed to result from a choriocarcinoma of the placenta that spreads to the child. Most infants present with a characteristic clinical picture of anaemia, hepatomegaly and precocious puberty. Imaging findings, including conventional MRI, may be nonspecific. To improve the accuracy of diagnosis, we present the imaging findings of contrast-enhanced dynamic MRI in a 4.5-month-old boy with infantile hepatic choriocarcinoma.
\end{abstract}

Keywords Liver · Neoplasm · Infantile choriocarcinoma . MRI · Child

\author{
M. van der Hoef · U. V. Willi \\ Department of Diagnostic Imaging, \\ University Children's Hospital Zurich, \\ Steinwiesstrasse 75, 8032 Zurich, \\ Switzerland \\ E-mail: thierry.huisman@kispi.unizh.ch \\ Tel.: + 41-1-2667110 \\ Fax: +41-1-2667158 \\ F. K. Niggli \\ Department of Paediatrics, \\ University Children's Hospital Zurich, \\ Zurich, Switzerland
}

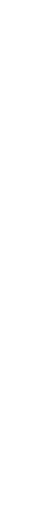




\section{Case report}

A 4.5-month-old boy was referred to our hospital because of a febrile urinary tract infection (UTI). Urinary analysis suggested UTI (leucocytes +++ , erythrocytes $+)$. Routine laboratory tests showed a significant microcytic hypochromic anaemia (red blood cell count $346 \times 10^{4} / \mathrm{ml}$, haemoglobin $5.2 \mathrm{~g} / \mathrm{dl}$, haematocrit $19 \%$, MCV $54 \mathrm{fl}, \mathrm{MCH} 15 \mathrm{pg}, \mathrm{MCHC} 277 \mathrm{~g} / \mathrm{l})$. Physical examination revealed signs of precocious puberty with an enlarged penis, increased testicular volume and pubic hair. There was no organomegaly. The child was otherwise healthy and had no previous history of severe illness. Pregnancy and birth were unremarkable.

Abdominal ultrasonagraphy (US) revealed a solitary $6.8 \times 5.0 \times 4.4 \mathrm{~cm}$, heterogeneous hypovascular mass within liver segments IVa and VIII. The remainder of the liver was normal. To improve tumour characterization, anatomical localization and to exclude additional lesions, dynamic contrast-enhanced MRI (1.5 T; Twin Speed, General Electric, Milwaukee, Wis., USA) was performed. Standard axial T1-weighted (T1) spin-echo (TR/TE 400/24, NEX 1, matrix 512×224, FOV $24 \times 18 \mathrm{~cm}$, slice thickness $6 \mathrm{~mm}$, interslice gap $1.2 \mathrm{~mm}$ ) and coronal T2-weighted (T2) fast spin-echo with fat suppression (TR/TE 8,333/110, NEX 2, matrix 320×224, FOV $26 \times 20 \mathrm{~cm}$, slice thickness $4 \mathrm{~mm}$, interslice gap $0.8 \mathrm{~mm}$ ) images were followed by a dynamic threedimensional T1 gradient-echo sequence (TR/TE 2.7/0.7, NEX 1, matrix $192 \times 128$, FOV $28 \times 20 \mathrm{~cm}$, slab thickness $64 \mathrm{~mm}$, slice thickness reconstruction $1.5 \mathrm{~mm}$ ).

A pre-contrast sequence was followed by three postcontrast acquisitions (arterial, porto-venous and delayed venous phase). Imaging was performed while the child was breathing spontaneously. Contrast medium (gadolinium $0.1 \mathrm{ml} / \mathrm{kg}$ body weight) was injected manually using a peripheral venous line. The well-circumscribed mass showed mixed T1- and T2- hypo- and hyperintense signal (Fig. 1). Within the centre of the lesion a focal T1 hyperintensity was seen indicating haemorrhage. After injection of a gadolinium-based contrast medium, the mass showed strong peripheral enhancement in the arterial phase that persisted into the portal venous and delayed venous phases (Fig. 2). The central areas showed minimal irregular enhancement without signs of filling-in on the delayed-phase scans (Fig. 3). No additional focal lesions or enlarged lymph nodes were seen.

The lesion was initially interpreted as either a hepatoblastoma or hemangioendothelioma. Further laboratory tests were negative for $\alpha$-fetoprotein and catecholamines. Liver function tests were within normal limits. Screening tests for hepatic infections were negative. The $\beta$-HCG level within the infant's serum was, however, distinctly elevated $(>40,000 \mathrm{U} / \mathrm{l})$. This laboratory finding in combination with the histological examination of a tumour biopsy established the diagnosis of solitary, infantile, malignant germ-cell tumour of the liver. Histology was compatible with choriocarcinoma with intense positivity for cytokeratin and $\beta$ HCG. Additional tumour work-up did not reveal any extrahepatic tumour spread. The child's mother has not yet been screened for $\beta$-HCG.

Neoadjuvant chemotherapy was administered for tumour reduction, followed by complete tumour resection. The child is currently in complete remission 7 months after diagnosis.

\section{Discussion}

Infantile hepatic choriocarcinoma is an extremely rare malignant tumour of the newborn and young infant. Fewer than 30 cases have been described. The clinical presentation is known as infantile choriocarcinoma
Fig. 1 A Axial, T1 spin-echo image shows a heterogeneous lesion with focal hyperintensity indicating haemorrhage in liver segments IVa and VIII. B Coronal, T2 fast spin-echo image. The lesion is T2- hypo- and hyperintense and appears well marginated. No additional intrahepatic lesions are identified
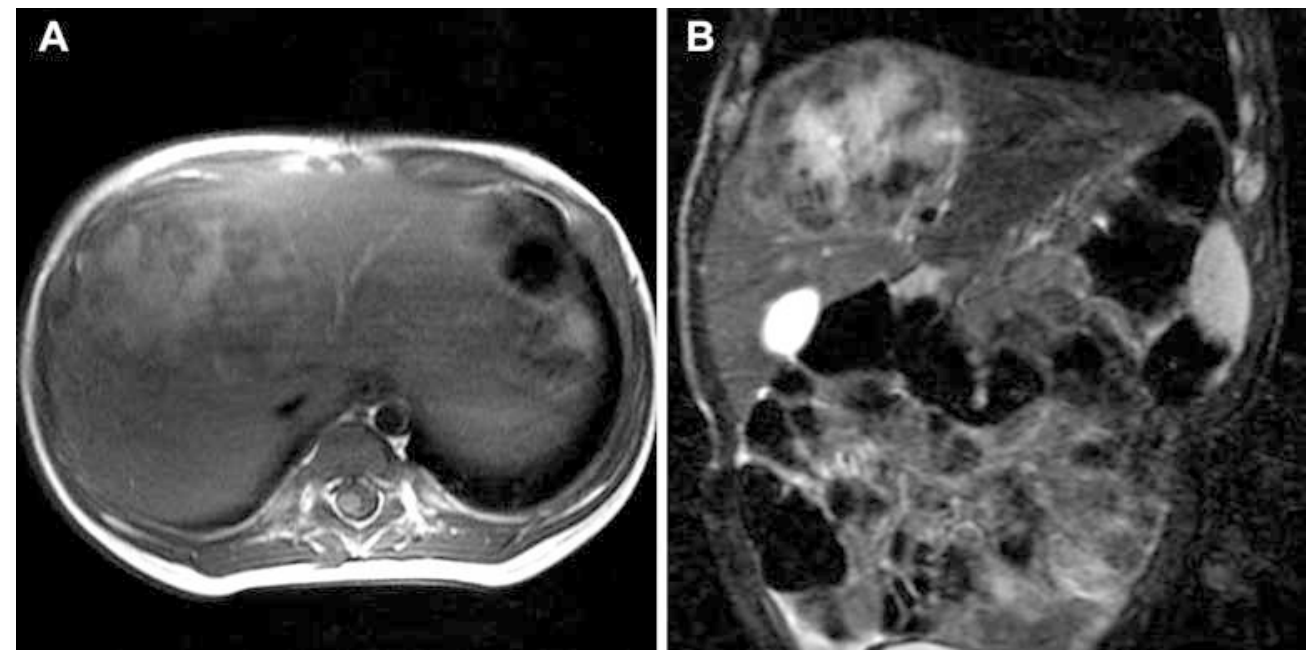


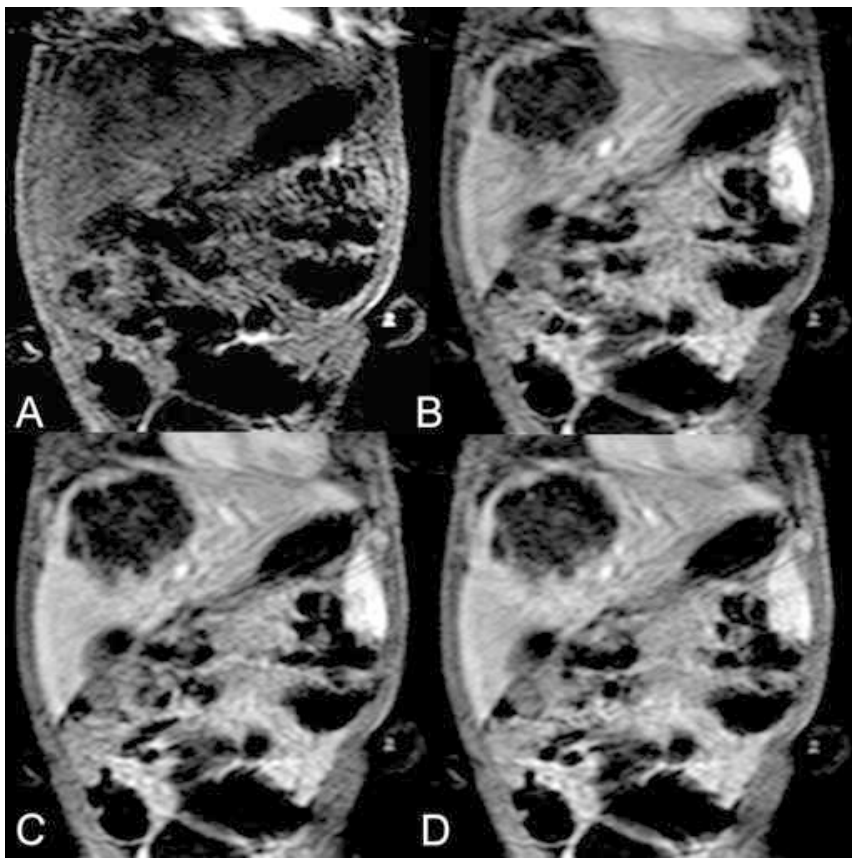

Fig. 2A-D Coronal, dynamic, contrast-enhanced, multiphase gradient-echo MRI. A The lesion is slightly hypointense to adjacent liver parenchyma on the pre-contrast sequence. B On the arterial phase the normal liver parenchyma and tumour periphery display strong contrast enhancement, whereas the central part of the tumour does not enhance. In the venous phase (C) and in the delayed venous phase (D) no significant delayed contrast enhancement of the central tumour parts is seen (no 'filling in')

syndrome', characterized by anaemia, hepatomegaly and precocious puberty [3]. The development of choriocarcinoma in an infant is often believed to be a complication of placental choriocarcinoma that metastasizes to the infant. The primary placental tumour is rarely diagnosed because the primary tumour in the placenta is usually too small to be recognized on

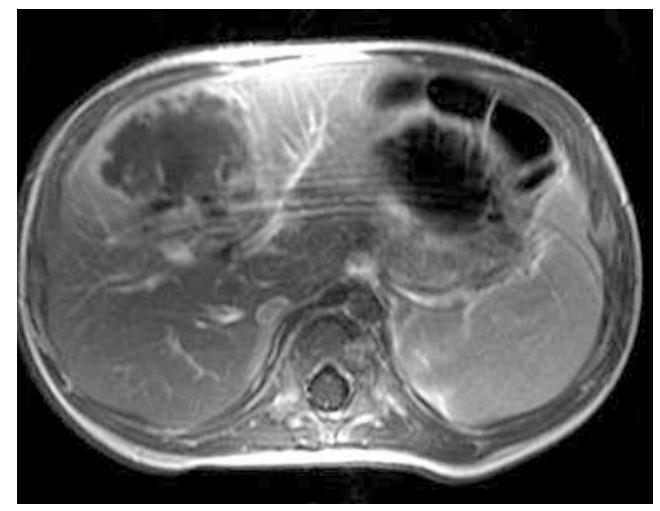

Fig. 3 Axial, delayed post-contrast, T1 spin-echo image. The central tumour areas show no contrast enhancement. There is no 'filling in' of the lesion inspection. Consequently, the placenta is rarely examined histologically. Also in our case, the placenta was no longer available at the time of diagnosis.

Choriocarcinoma of the placenta has an incidence of 1 in 50,000 pregnancies [2]. Most infantile cases of choriocarcinoma occur between 5 weeks and 7 months of age or at term [1, 5]. Early dissemination and intractable disease require rapid diagnosis and treatment $[1,4]$. Common sites of metastases for infantile hepatic choriocarcinoma are lung, kidney, brain and lymph nodes. The principal specific diagnostic test is the measurement of $\beta$-HCG levels in the serum and/or urine, which will be markedly elevated in the presence of the tumour. $\beta$-HCG is normally secreted by the placenta and by gonadal tumours containing trophoblastic cells $[2,7-9]$. Elevated $\beta$-HCG levels cause the precocious puberty. The decline in serial serum $\beta$-HCG levels can be used to monitor treatment response. The tumour is usually highly susceptible to chemotherapy [10].

Few studies have described the imaging findings of infantile hepatic choriocarcinoma $[1,2,4,5]$. Although MRI is very sensitive for tumour detection and gives detailed anatomical information, the signal characteristics are non-specific. Choriocarcinoma usually has heterogeneous T1- and T2-signal intensity with large areas of central necrosis. T1-hyperintense haemorrhagic areas are frequently encountered, in addition to fluid-filled cysts with high-protein content that are T1- and T2hyperintense. The pattern of contrast enhancement is also not very specific and does not improve diagnostic accuracy. Choriocarcinomas usually show strong and irregular peripheral enhancement without central tumoural enhancement. Consequently, in the absence of a known maternal choriocarcinoma, the tumour is usually misdiagnosed as hepatoblastoma, hemangioendothelioma, mesenchymal hamartoma, metastatic neuroblastoma or as undifferentiated hepatic sarcoma $[2,4$ 6].

To the best of our knowledge, this report is the first to describe the enhancement pattern on dynamic gadolinium-enhanced MRI of an infantile hepatic choriocarcinoma. The tumour showed strong peripheral enhancement in the arterial phase that persisted into the delayed venous phase. There was no central filling-in of the tumour. On delayed-phase images obtained $5 \mathrm{~min}$ after contrast injection, there was no significant enhancement of the central tumour areas. This contrast pattern clearly differentiates the tumour from infantile hemangioendothelioma [6]. The absence of elevated $\alpha$-fetoprotein and vanillylmandelic acid strongly militates against hepatoblastoma and neuroblastoma. Mesenchymal hamartoma usually shows more solid, contrastenhancing components. The contrast-enhancement pattern of the presented choriocarcinoma does not allow differentiation from undifferentiated hepatic sarcoma. The clinically observed signs of precocious puberty and 
anaemia, together with elevated $\beta$-HCG levels are, however, strongly indicative of choriocarcinoma.

In conclusion, this case shows that the MR signal intensities and dynamic contrast enhancement pattern are not characteristic for choriocarcinoma. The lack of filling-in on delayed sequences differentiates it from hemangioendothelioma that may look very similar on T1 and T2 MRI sequences. The specific diagnostic test is measurement of serum and/or urine $\beta$-HCG. Catecholamines and $\alpha$-fetoprotein should be estimated to exclude neuroblastoma and hepatoblastoma, respectively. It should be remembered that infantile choriocarcinoma may later disseminate in the mother and she should also be screened for elevated $\beta$-HCG levels.

\section{References}

1. Szavay PO, Wermes C, Fuchs J, et al (2000) Effective treatment of infantile choriocarcinoma in the liver with chemotherapy and surgical resection: a case report. J Pediatr Surg 35:1134-1135

2. Kishkurno S, Ishida A, Takahashi Y, et al (1997) A case of neonatal choriocarcinoma. Am J Perinatol 14:79-82

3. Witzleben CL, Bruninga G (1968) Infantile choriocarcinoma: a characteristic syndrome. J Pediatr 73:374-378
4. Sashi R, Sato K, Hirano H, et al (1996) Infantile choriocarcinoma: a case report with MRI, angiography and bone scintigraphy. Pediatr Radiol 26:869-870

5. Moon WK, Kim WS, Kim I, et al (1993) Hepatic choriocarcinoma in a neonate: MR appearance. J Comput Assist Tomogr 17:653-655

6. Mortele KJ, Vanzieleghem B, Mortele B et al (2002) Solitary hepatic infantile hemangioendothelioma: dynamic gadolinium-enhanced MR imaging findings. Eur Radiol 12:862-865

7. Chou H, Chen R, Yau KT, et al (2002) Infantile choriocarcinoma with idiopathic massive fetomaternal hemorrhage. Med Pediatr Oncol 38:203-204
8. Blohm ME, Calaminus G, Gnekow AK, et al (2001) Disseminated choriocarcinoma in infancy is curable by chemotherapy and delayed tumour resection. Eur J Cancer 37:72-78

9. Andreitchouk AE, Takahashi O, Kodama H, et al (1996) Choriocarcinoma in infant and mother: a case report. J Obstet Gynaecol Res 22:585-588

10. Johnson EJ, Crofton PM, O`Neill JM, et al (2003) Infantile choriocarcinoma treated with chemotherapy alone. Med Pediatr Oncol 41:550-557 